\title{
O-SPECIES AND TENSOR ALGEBRAS
}

\author{
Nadiya Gubareni \\ Institute of Mathematics, Czestochowa University of Technology \\ Częstochowa, Poland \\ nadiya.gubareni@yahoo.com
}

\begin{abstract}
In this paper we consider $O$-species and their representations. These $O$-species are a type of a generalization of a species introduced by Gabriel. We also consider the tensor algebras of such $O$-species. It is proved that the category of all representations of an $O$-species and the category of all right modules over the corresponding tensor algebra are naturally equivalent.
\end{abstract}

Keywords: species, $O$-species, representations of O-species, tensor algebra, O-species of bounded representation type, diagram of O-species

\section{Introduction}

In this paper we consider $O$-species, which generalize the notion of species introduced by Gabriel in [1]. Recall this definition:

Definition 1.1. (Gabriel [1]). Let $I$ be a finite index set. A species $L=\left(F_{i},{ }_{i} M_{j}\right)_{i, j \in I}$ is a finite family $\left(F_{i}\right)_{i \in I}$ of division rings together with a family $\left({ }_{i} M_{j}\right)_{i, j} \in I$ of $\left(F_{i}, F_{j}\right)$-bimodules.

We say that $\left(F_{i,}{ }_{i} M_{j}\right)_{i, j \in I}$ is a $\boldsymbol{K}$-species if all $F_{i}$ are finite dimensional and central over the common commutative subfield $K$ which acts centrally on ${ }_{i} M_{i}$, i.e. $\lambda m=m \lambda$ for all $\lambda \in K$ and all $m \in{ }_{i} M_{j}$. We also assume that each bimodule ${ }_{i} M_{j}$ is a finite dimensional vector space over $K . K$-species is a $K$-quiver if $F_{i}=K$ for each $i$.

Definition 1.2. A representation $\left(V_{i,}, \varphi_{i}\right)$ of a species $L=\left(F_{i,}{ }_{i} M_{j}\right)_{i, j} \in I$ (or an $\boldsymbol{L}$-representation) is a family of right $F_{i}$-modules $V_{i}$ and $F_{j}$-linear mappings:

$$
{ }_{j} \varphi_{i}: V_{i} \otimes_{F_{i}} M_{j} \rightarrow V_{j}
$$

for each $i, j \in I$. Such a representation is called finite dimensional, provided all the spaces $V_{i}$ are finite dimensional vector spaces. 
Let $V=\left(V_{i},{ }_{j} \varphi_{i}\right)$ and $W=\left(W_{i},{ }_{j} \psi_{i}\right)$ be two $L$-representations. An $L$-morphism $\Psi$ : $V \rightarrow W$ is a set of $F_{i}$-linear maps $\alpha_{i}: V_{i} \rightarrow W_{i}$ such that

$$
{ }_{j} \psi_{i}\left(\alpha_{i} \otimes 1\right)=\alpha_{j} \cdot{ }_{j} \varphi_{i}
$$

Two representations $\left(V_{i},{ }_{j} \varphi_{i}\right)$ and $W=\left(W_{i, j} \psi_{i}\right)$ are called equivalent if there is a set of isomorphisms $\alpha_{i}$ from the $F_{i}$-module $V_{i}$ to the $F_{i}$-module $W_{i}$ such that the (1.4) holds for all $i, j \in I$.

A representation $\left(V_{i},{ }_{j} \varphi_{i}\right)$ is called indecomposable, if there are no non-zero sets of subspaces $\left(U_{i}\right)$ and $\left(W_{i}\right)$ such that $V_{i}=U_{i} \oplus W_{i}$ and ${ }_{j} \varphi_{i}={ }_{j} \psi_{i} \oplus_{j} \tau_{i}$, where

$$
\begin{gathered}
{ }_{j} \psi_{i}: U_{i} \otimes_{F_{i}}{ }_{i} M_{j} \rightarrow U_{j} \\
{ }_{j} \tau_{i}: W_{i} \otimes_{F_{i}}{ }_{i} M_{j} \rightarrow W_{j}
\end{gathered}
$$

One defines the direct sum of two $L$-representations in the obvious way.

Denote by $\operatorname{Rep}(L)$ the category of all $L$-representations, and by $\operatorname{rep}(L)$ the category of finite dimensional $L$-representations, whose objects are $L$-representations and whose morphisms are as defined above.

Definition 1.7. [2] A species $L=\left(F_{i},{ }_{i} M_{j}\right)_{i, j \in I}$ is said to be of finite type, if the number of indecomposable non-isomorphic finite dimensional representations is finite.

A species $L=\left(F_{i},{ }_{i} M_{j}\right)_{i, j \in I}$ is said to be of strongly unbounded type if it possesses the following three properties:

1. $L$ has indecomposable objects of arbitrary large finite dimension.

2. If $L$ contains a finite dimensional object with an infinite endomorphism ring, then there is an infinite number of (finite) dimensions $d$ such that, for each $d$, the species $L$ has infinitely many (non-isomorphic) indecomposable objects of dimension $d$.

3. $L$ has indecomposable objects of infinite dimension.

Dlab and Ringel proved in [2, Theorem E] that any $K$-species is either of finite or of strongly unbounded type.

With any species $L=\left(F_{i},{ }_{i} M_{j}\right)_{i, j \in I}$ one can define the tensor algebra in the following way. Let $B=\prod_{i \in I} F_{i}$, and let $M=\bigoplus_{i, j \in I} M_{j}$. Then $B$ is a ring and $M$ naturally becomes a $(B, B)$-bimodule. The tensor algebra of the $(B, B)$-bimodule $M$ is the graded ring 


$$
T(L)=T_{B}(M)=\bigoplus_{n=0}^{\infty} M^{\otimes n}
$$

with component-wise addition and the multiplication induced by taking tensor products.

If $L$ is a $K$-species, then $T(L)$ is a finite dimensional $K$-algebra.

Theorem 1.9. (Dlab, Ringel [2, Proposition 10.1]). Let $L$ be a $K$-species. Then the category $\operatorname{Rep}(L)$ of all representations of $L$ and the category $\operatorname{Mod}_{r}(T(L))$ of all right $T(L)$-modules are equivalent.

\section{2. $O$-species and their representations}

In this section we consider the notion of $O$-species, which generalizes the notion of species considered in [1].

Let $\left\{O_{i}\right\}$ be a family of discrete valuation rings (not necessarily commutative) $O_{i}$ with radicals $R_{i}$ and skew fields of fractions $D_{i}$, for $i=1,2, \ldots, k$, and let $\left\{D_{j}\right\}$, for $j=k+1, \ldots, n$, be a family of skew fields. Let $\left(n_{1}, n_{2}, \ldots, n_{k}\right)$ be a set of natural numbers. Write

$$
H_{n_{i}}\left(O_{i}\right)=\left(\begin{array}{cccc}
O_{i} & O_{i} & \cdots & O_{i} \\
R_{i} & O_{i} & \cdots & O_{i} \\
\vdots & \vdots & \ddots & \vdots \\
R_{i} & R_{i} & \cdots & O_{i}
\end{array}\right)
$$

which is a subring in the matrix ring $M_{n_{i}}\left(D_{i}\right)$. It is easy to see that each $H_{n_{i}}\left(O_{i}\right)$ is a Noetherian serial prime hereditary ring. Write $F_{i}=H_{n_{i}}\left(O_{i}\right)$ for $i=1,2, \ldots, k$, and $F_{j}=D_{j}$ for $j=k+1, \ldots, n$. Then, by the Goldie theorem, there exists a classical ring of fractions $\widetilde{F}_{i}$ for $i=1,2, \ldots, n$.

Consider the following generalization of a species.

Definition 2.1. An $\boldsymbol{O}$-species is a set $\Omega=\left(F_{i},{ }_{i} M_{j}\right)_{i, j} \in I$, where $F_{i}=H_{n_{i}}\left(O_{i}\right)$ for $i=1,2, \ldots, k$, and $F_{j}=D_{j}$ for $j=k+1, \ldots, n$, and moreover ${ }_{i} M_{j}$ is an $\left(\widetilde{F}_{i}, \widetilde{F}_{j}\right)$ bimodule, which is finite dimensional as a right $D_{j}$-vector space and as a left $D_{i}$-vector space.

An $O$-species $\Omega$ is called a $(\boldsymbol{D}, \boldsymbol{O})$-species if all $O_{i}$ have a common skew field of fractions $D$, i.e. all $D_{i}$ are equal to a fixed skew field $D$ and 


$$
{ }_{D}\left({ }_{i} M_{j}\right)_{D} \cong\left({ }_{D} D_{D}\right)^{n_{i j}}
$$

for some natural number $n_{i j}(i=1,2, \ldots, n)$.

An $O$-species $\Omega$ is called a $(\boldsymbol{K}, \boldsymbol{O})$-species, if all $D_{i}(i=1,2, \ldots, n)$ contain a common central subfield $K$ of finite index in such a way that $\lambda m=m \lambda$ for all $\lambda \in K$ and all $m \in{ }_{i} M_{j}$ (moreover, each bimodule ${ }_{i} M_{j}$ is a finite dimensional vector space over $K)$. It is a $(K, O)$-quiver if moreover $D_{i}=D$ for each $i$.

Everywhere in this paper we will consider $O$-species without oriented cycles and loops, i.e. we will assume that ${ }_{i} M_{i}=0$, and if ${ }_{i} M_{j} \neq 0$, then ${ }_{j} M_{i}=0$. A vertex $i$ is said to be marked if $F_{i}=H_{n_{i}}\left(O_{i}\right)$.

We will also assume that all marked vertices are minimal, i.e. ${ }_{j} M_{i}=0$ if $F_{i}=H_{n_{i}}\left(O_{i}\right)$, and that ${ }_{i} M_{j}={ }_{j} M_{i}=0$ if $i, j$ are marked vertices.

Definition 2.3. The diagram of an $O$-species $\Omega=\left\{F_{i},{ }_{i} M_{j}\right\}_{i, j \in I}$ is defined in the following way:

1. The set of vertices is a finite set $I=\{1,2, \ldots, n\}$.

2. The finite subset $I_{0}=\{1,2, \ldots, k\}$ of $I$ is a set of marked points.

3 . The vertex $i$ connects with the vertex $j$ by $t_{i j}$ arrows, where

$$
t_{i j}=\frac{1}{n_{i}} \operatorname{dim}_{D}\left({ }_{i} M_{j}\right) \times \operatorname{dim}\left({ }_{i} M_{j}\right)_{D}+\frac{1}{n_{j}} \operatorname{dim}_{D}\left({ }_{j} M_{i}\right) \times \operatorname{dim}\left({ }_{j} M_{i}\right)_{D}
$$

moreover, we assume that $n_{i}=1$ if $F_{i}=D_{i}$.

Similar to species we can define representations of $O$-species in the following way.

Definition 2.4. A representation $\left(M_{i}, V_{r},{ }_{j} \varphi_{i},{ }_{j} \psi_{r}\right)$ of an $O$-species $\Omega=\left\{F_{i,}{ }_{i} M_{j}\right\}_{i, j \in I}$ is a family of right $F_{i}$-modules $M_{i}(i=1,2, \ldots, k)$, a set of right vector spaces $V_{r}$ over $D_{r}(r=k+1, k+1, \ldots, n)$ and $D_{j}$-linear maps:

$$
{ }_{j} \varphi_{i}: M_{i} \otimes_{F_{i} i} M_{j} \rightarrow V_{j}
$$

for each $i=1,2, \ldots, k ; j=k+1, k+2, \ldots, n$; and

$$
{ }_{j} \psi_{r}: V_{r} \otimes_{D_{r} r} M_{j} \rightarrow V_{j}
$$

for each $r, j=k+1, k+2, \ldots, n$.

Definition 2.5. Two representations $M=\left(M_{i}, V_{r},{ }_{j} \varphi_{i, j} \psi_{r}\right)$ and $M^{\prime}=\left(M_{i}^{\prime}, V_{r}^{\prime},{ }_{j} \varphi_{i}^{\prime},{ }_{j} \psi_{r}^{\prime}\right)$ are called equivalent if there is a set of isomorphisms $\alpha_{i}$ of $F_{i}$-modules from $M_{i}$ to 
$M_{i}^{\prime}$ and a set of isomorphisms $\beta_{r}$ of $D_{r}$-vector spaces from $V_{r}$ to $V_{r}^{\prime}$ such that for each $i=1,2, \ldots, k ; r, j=k+1, k+2, \ldots, n$ the following equalities hold:

$$
\begin{gathered}
{ }_{j} \varphi_{i}^{\prime}\left(\alpha_{i} \otimes 1\right)=\beta_{j} \cdot{ }_{j} \varphi_{i} \\
{ }_{j} \psi_{r}^{\prime}\left(\beta_{r} \otimes 1\right)=\beta_{j} \cdot{ }_{j} \psi_{r}
\end{gathered}
$$

In a natural way one can define the notions of a direct sum of representations and of an indecomposable representation.

The set of all representations of an $O$-species $\Omega=\left(F_{i},{ }_{i} M_{j}\right)_{i, j \in I}$ can be turned into a category $R(\Omega)$, whose objects are representations $M=\left(M_{i}, V_{r},{ }_{j} \varphi_{i},{ }_{j} \psi_{r}\right)$, and a morphism from object $M=\left(M_{i}, V_{r},{ }_{j} \varphi_{i},{ }_{j} \psi_{r}\right)$ to object $M^{\prime}=\left(M_{i}^{\prime}, V_{r}^{\prime},{ }_{j} \varphi_{i}^{\prime},{ }_{j} \psi_{r}^{\prime}\right)$ is a set of homomorphisms $\alpha_{i}$ of $H_{n_{i}}\left(O_{i}\right)$ - modules $M_{i}$ to $M_{i}^{\prime}$, and a set of homomorphisms $\beta_{r}$ of $D_{r}$ - vector spaces from $V_{r}$ to $V_{r}^{\prime}$ such that for each $i=1,2, \ldots, k$; $r, j=k+1, k+2, \ldots, n$ the equalities (2.6) and (2.7) hold.

\section{Tensor algebra of $\mathrm{O}$-species}

For any $O$-species $\Omega=\left(F_{i},{ }_{i} M_{j}\right)_{i, j \in I}$ one can construct a tensor algebra of bimodules $T(\Omega)$. Let $A=\bigoplus_{i=1}^{n} F_{i}, B=\bigoplus_{i, j} M_{j}$. Then $B$ is an $(A, A)$ - bimodule and we can define a tensor algebra $T_{A}(B)$ of the bimodule $B$ over the ring $A$ in the following way:

$$
T_{A}(B)=A \oplus B \oplus B^{2} \oplus \ldots \oplus B^{n} \oplus \ldots
$$

is a graded ring, where $B^{n}=B \otimes_{A} B^{n-1}$ for $n>1$, and multiplication in $T_{A}(B)$ is given by the natural $A$-bilinear map:

$$
B^{n} \times B^{m} \rightarrow B^{n} \otimes_{A} B^{m}=B^{n+m}
$$

Then $T(\Omega)=T_{A}(B)$ is the tensor algebra corresponding to an $O$-species $\Omega$.

Proposition 3.3. Let $\Omega$ be an $O$-species. Then the category $\Re(\Omega)$ of all representations of $\Omega$ and the category $\operatorname{Mod}_{\mathrm{r}} T(\Omega)$ of all right $T(\Omega)$-modules are naturally equivalent.

Proof. Form two functors $R: \operatorname{Mod}_{\mathrm{r}} T(\Omega) \rightarrow \mathfrak{R}(\Omega)$ and $P: \mathfrak{R}(\Omega) \rightarrow \operatorname{Mod}_{\mathrm{r}} T(\Omega)$ in the following way. Let $X_{T(\Omega)}$ be a right $T(\Omega)$-module. Since $A$ is a subring in $T(\Omega), X$ can be considered as a right $A$-module. Then 


$$
X=\left(\bigoplus_{i=1}^{k} M_{i}\right) \oplus\left(\bigoplus_{r=k+1}^{n} V_{r}\right),
$$

where $M_{i}$ is an $H_{n_{i}}\left(O_{i}\right)$-module, and $V_{r}$ is a $D_{r}$-vector space; moreover, $M_{i} H_{n_{j}}\left(O_{j}\right)=0$ for $i \neq j$, and $V_{r} D_{s}=0$ for $r \neq s$. Since $B$ is an $(A, A)$-bimodule, one can define an $A$-homomorphism $\varphi: X \otimes{ }_{A} B \rightarrow X_{A}$. Taking into account that $M_{i} \otimes_{A}{ }_{s} M_{j}=0$ for $i \neq s$, the map $\varphi$ is defined in the following way:

$$
\varphi:\left(\bigoplus_{i=1}^{k}\left(M_{i} \otimes_{A i} M_{j}\right)\right) \oplus\left(\bigoplus_{r=k+1}^{n}\left(V_{r} \otimes_{A} M_{j}\right)\right) \rightarrow \bigoplus_{r=k+1}^{n} V_{r}
$$

Since $M_{i} \otimes_{A}{ }_{i} M_{j}$ is mapping into $V_{j}$, and $V_{r} \otimes_{A}{ }_{r} M_{j}$ is mapping into $V_{j}, \varphi$ defines a set of $D_{j}$-homomorphisms:

$$
\begin{gathered}
{ }_{j} \varphi_{i}: M_{i} \otimes_{A} M_{j}=M_{i} \otimes_{H_{n_{i}\left(O_{i}\right)}{ }_{i}} M_{j} \rightarrow V_{j} \\
{ }_{j} \psi_{r}: V_{r} \otimes_{A}{ }_{r} M_{j}=V_{r} \otimes_{D_{r} r} M_{j} \rightarrow V_{j}
\end{gathered}
$$

for $i=1,2, \ldots, k ; r, j=k+1, \ldots, n$.

Now one can define $R\left(X_{T(\Omega)}\right)=\left(M_{i}, V_{r},{ }_{j} \varphi_{i},{ }_{j} \psi_{r}\right)$. Let $X, Y$ be two right $T(\Omega)$-modules, let $\alpha: X \rightarrow Y$ be a homomorphism, and let $R(X)=\left(M_{i}, V_{r},{ }_{j} \varphi_{i},{ }_{j} \psi_{r}\right)$, $R(Y)=\left(N_{i}, W_{r},{ }_{j} \widetilde{\varphi}_{i},{ }_{j} \widetilde{\psi}_{r}\right)$. Let's define a morphism from $R(X)$ to $R(Y)$. Since $\alpha$ is an $A$-homomorphism, $\alpha\left(M_{i}\right) \subseteq N_{i}, \alpha\left(V_{r}\right) \subseteq W_{r}$, i.e., $\alpha$ defines a family of $H_{n_{i}}\left(O_{i}\right)$-homomorphisms $\alpha_{i}: M_{i} \rightarrow N_{i}$ and a family of $D_{r}$-homomorphisms $\beta_{r}$ : $V_{r} \rightarrow W_{r}$, which are the restrictions of $\alpha$ to $M_{i}$ and $V_{r}$. Therefore one can set $R(\alpha)=\left\{\left(\alpha_{i}\right),\left(\beta_{r}\right)\right\}$. Since $\alpha$ is a $T(\Omega)$-homomorphism,

$$
{ }_{j} \widetilde{\varphi}_{i}\left(\alpha_{i} \otimes 1\right)=\alpha_{j} \cdot{ }_{j} \varphi_{i}
$$

and

$$
{ }_{j} \widetilde{\psi}_{r}\left(\beta_{r} \otimes 1\right)=\beta_{j} \cdot{ }_{j} \psi_{r}
$$

for $i=1,2, \ldots, k ; r, j=k+1, \ldots, n$. Therefore $R(\alpha)$ is a morphism in the category $R(\Omega)$.

Conversely, let $\Omega=\left(F_{i},{ }_{i} M_{j}\right)_{i, j \in I}$ and there is given a representation $M=\left(M_{i}, V_{r}\right.$, $\left.{ }_{j} \varphi_{i},{ }_{j} \psi_{r}\right)$. Then one can define $P(M)$ in the following way:

$$
P(M)=X=\left(\bigoplus_{i=1}^{k} M_{i}\right) \oplus\left(\bigoplus_{r=k+1}^{n} V_{r}\right) .
$$


We define an action of

$$
A=\left(\bigoplus_{i=1}^{k} H_{n_{i}}\left(O_{i}\right)\right) \oplus\left(\bigoplus_{r=k+1}^{n} D_{r}\right)
$$

on $M_{i}$ by means of the projection $A \rightarrow H_{n_{i}}\left(O_{i}\right)$ and an action of $A$ on $V_{r}$ by means of the projection $A \rightarrow D_{r}$. We define an action of $B^{n}$ on $X$ by induction of $\varphi^{(n)}: X \otimes_{A} B^{n} \rightarrow X$ as follows:

$$
\begin{aligned}
\varphi^{(1)} & =\bigoplus_{i, j} \varphi_{j} \bigoplus_{j, r} \psi_{r}: X \otimes_{A} B=\left(\bigoplus_{i=1}^{k}\left(M_{i} \otimes_{A} M_{j}\right)\right) \oplus\left(\bigoplus_{r=k+1}^{n}\left(V_{r} \otimes_{A}{ }_{r} M_{j}\right)\right)= \\
& =\left(\bigoplus_{i=1}^{k}\left(M_{i} \otimes_{H_{n_{i}\left(O_{i}\right)}} M_{j}\right)\right) \oplus\left(\bigoplus_{r=k+1}^{n}\left(V_{r} \otimes_{D_{r} r} M_{j}\right)\right) \rightarrow \bigoplus_{r=k+1}^{n} V_{r} \subseteq X . \\
\varphi^{(n+1)} & =\varphi\left(\varphi^{(n)} \otimes 1\right): X \otimes_{A} B^{(n+1)}=\left(X \otimes_{A} B\right) \otimes_{A} B^{n} \stackrel{\varphi^{(n)} \otimes 1}{\rightarrow} X \otimes_{A} B \stackrel{\varphi}{\rightarrow} X
\end{aligned}
$$

If $\alpha=\left\{\left\{\alpha_{i}\right\},\left\{\beta_{r}\right\}\right\}$ is a morphism of a representation $M=\left(M_{i}, V_{r},{ }_{j} \varphi_{i},{ }_{j} \psi_{r}\right)$ to a representation $M^{\prime}=\left(M_{i}^{\prime}, V_{r}^{\prime},{ }_{j} \varphi_{i}^{\prime},{ }_{j} \psi_{r}^{\prime}\right), X=P(M), Y=P\left(M^{\prime}\right)$, then

$$
\varphi=\bigoplus_{i} \alpha_{i} \bigoplus_{r} \beta_{r}: X=\bigoplus_{i} M_{i} \bigoplus_{r} V_{r} \rightarrow \bigoplus_{i} M_{i}^{\prime} \bigoplus_{r} V_{r}^{\prime}
$$

is a $T(\Omega)$-homomorphism and therefore $P(\alpha)=\varphi$.

It is not difficult to show that $R, P$ are mutually inverse functors and they give an equivalence of categories $\operatorname{Mod}_{\mathrm{r}} T(\Omega)$ and $\mathfrak{R}(\Omega)$.

Recall that an Artinian $\operatorname{ring} A$ is of finite representation type if $A$ has only a finite number of indecomposable finitely generated right $A$-modules up to isomorphism.

A ring $A$ is of (right) bounded representation type (see $[3,4]$ ) if there is an upper bound on the number of generators required for indecomposable finitely presented right $A$-modules.

Denote by $\mu\left(M_{i}\right)$ the minimal number of generators of an $H_{n_{i}}\left(O_{i}\right)$-module $M_{i}$, and denote by $d_{r}=\operatorname{dim}_{D_{r}}\left(V_{r}\right)$ the dimension of vector space $V_{r}$ over $D_{r}$. The dimension of a representation $M=\left(M_{i}, V_{r},{ }_{j} \varphi_{i, j} \psi_{r}\right)$ is the number

$$
d=\operatorname{dim} M=\sum_{i=1}^{n} \mu\left(M_{i}\right)+\sum_{r=k+1}^{n} d_{r}
$$


Definition 3.14. An $O$-species $\Omega$ is said to be of bounded representation type if the dimensions of its indecomposable finite dimensional representations have an upper bound.

Corollary 3.15. An $O$-species $\Omega$ is of bounded representation type if and only if the tensor algebra $T(\Omega)$ is of bounded representation type.

Proof. If $\Omega$ is an $O$-species of bounded representation type, then there exists $N>0$ such that $\operatorname{dim} M<N$ for any indecomposable finite dimensional representation $M$. Then for any finitely generated $T(\Omega)$-module $X$ we have $\mu(X)<N_{1}$, where $N_{1}$ is some fixed number depending on $N$, i.e. $T(\Omega)$ is a ring of bounded representation type. The converse also holds: if $T(\Omega)$ is a ring of bounded representation type, then $\Omega$ is an $O$-species of bounded representation type.

Corollary 3.16. Let $\Omega_{1}$ be a $D$-species, which is a subspecies of a $(D, O)$-species $\Omega$. If $\Omega$ is of bounded representation type, then $\Omega_{1}$ is of finite type.

Proof. Since $\Omega$ is of bounded representation type, each of its subspecies is of bounded representation type as well. So $\Omega_{1}$ is of bounded representation type, and, by corollary 3.15 , its tensor algebra is of bounded representation type, as well. Since $\Omega_{1}$ is a $D$-species, its tensor algebra is an Artinian ring. So it is of finite representation type, by [5]. Therefore, $\Omega_{1}$ is also of finite representation type.

\section{Conclusion}

In this paper we introduced $O$-species and the tensor algebras corresponding to them. These $O$-species are some generalizations of species first introduced by Gabriel in [1]. We consider the notion of a representation of an $O$-species. In this paper we prove that the category of all representations of $O$-species $\Omega$ and the category of all right modules over a tensor algebra $T(\Omega)$ are naturally equivalent.

\section{References}

[1] Gabriel P., Indecomposable representations I, Manuscripta Math. 1972, 6, 71-103.

[2] Dlab V., Ringel C.M., On algebras of finite representation type, Journal of Algebra 1975, 33, 306-394.

[3] Warfield R.B., Serial rings and finitely presented modules, Journal of Algebra 1975, 37, 2, $187-222$.

[4] Gubareni N., On right hereditary SPSD-rings of bounded representation type I, Scientific Research of the Institute of Mathematics and Computer Science 2012, 3(11), 57-70.

[5] Auslander M., Representation theory of Artin algebras II, Comm. Algebra 1974, 1, 269-310. 